\title{
Spitzer Observations of M83 and the Hot Star, H II Region Connection
}

\author{
R. H. Rubin ${ }^{1}$, J. P. Simpson ${ }^{1}$, S. W. J. Colgan ${ }^{1}$, R. J. Dufour ${ }^{2}$, \\ K. L. Ray $^{1}$, B. F. Wakefield ${ }^{1}$, D. B. Key ${ }^{1}$, E. F. Erickson ${ }^{1}$, \\ M. R. Haas ${ }^{1}$, and A. W. A. Pauldrach ${ }^{3}$ \\ ${ }^{1}$ NASA/Ames \\ ${ }^{2}$ Rice University \\ ${ }^{3}$ University Munich
}

\begin{abstract}
Emission-line observations made with the Spitzer Space Telescope are used to test the predicted ionizing spectral energy distributions of various stellar atmosphere models.
\end{abstract}

Keywords. ISM: abundances, HII regions, stars: atmospheres, galaxies: individual (M83)

\section{Introduction}

$\mathrm{H}$ II regions play a crucial role in the measurement of current interstellar abundances. Like planetary nebulae, they also serve as laboratories for atomic physics and provide fundamental data about heavy element abundances that constrain models of galactic chemical evolution. In 2005, February and July, we observed emission lines of [S IV] 10.5, [Ne II] 12.8, [Ne III] 15.6, \& [S III] $18.7 \mu \mathrm{m}$ cospatially with the Spitzer Space Telescope using the Infrared Spectrograph (IRS) in short-high mode (SH). From the measured fluxes, we determine the ionic abundance ratios $\mathrm{Ne}^{++} / \mathrm{Ne}^{+}, \mathrm{S}^{+3} / \mathrm{S}^{++}$, and $\mathrm{S}^{++} / \mathrm{Ne}^{+}$in $24 \mathrm{H}$ II regions in the nearly face-on spiral galaxy M83. We covered a range of deprojected galactocentric radii $\left(\mathrm{R}_{G}\right)$ from 0.46 to $5.16 \mathrm{kpc}$. Important advantages compared with prior optical studies are: 1) the IR lines have a weak and similar electron temperature $\left(T_{e}\right)$ dependence, while optical lines vary exponentially with $T_{e}$ and 2) the IR lines suffer far less from interstellar extinction. We examine the variation of $\mathrm{S}^{++} / \mathrm{Ne}^{+}$and $\mathrm{Ne}^{++} / \mathrm{Ne}^{+}$ with $\mathrm{R}_{G}$. The data constrain the ionizing spectral energy distribution (SED) for the stars exciting these nebulae. We compare the ionic ratios with predictions made using stellar atmosphere models from several different LTE and non-LTE model sets.

\section{Variation of ionic ratios with $\mathbf{R}_{G}$}

We find a clear correlation with $\mathrm{R}_{G}$ of the ionic abundance ratio $\mathrm{S}^{++} / \mathrm{Ne}^{+}$, which is derived from the measured line flux ratios for each $\mathrm{H}$ II region. For $\mathrm{H}$ II regions in M83, an electron density $\left(N_{e}\right)$ of $100 \mathrm{~cm}^{-3}$ is used as a typical value; an upper limit is roughly $1000 \mathrm{~cm}^{-3}$. For the lower $N_{e}$, a linear least-squares fit yields $\left\langle\mathrm{S}^{++}\right\rangle /\left\langle\mathrm{Ne}^{+}\right\rangle=$ $0.426+0.0347 \mathrm{R}_{G}$ (with $\mathrm{R}_{G}$ in $\mathrm{kpc}$ ), where angular brackets denote fractional ionization. The least-squares fit for an assumed $N_{e}=1000 \mathrm{~cm}^{-3}$ is $\left\langle\mathrm{S}^{++}\right\rangle /\left\langle\mathrm{Ne}^{+}\right\rangle=$ $0.365+0.0297 \mathrm{R}_{G}$. In the linear fits, we have assumed an Orion Nebula Ne/S abundance ratio of 14.3 (Simpson et al. 2004). Because Ne and S are "primary" elements, their production is expected to vary in lockstep and Ne/S would not be expected to show a radial gradient within a galaxy (Pagel \& Edmunds 1981). The fit to the ionic abundance ratio $\mathrm{Ne}^{++} / \mathrm{Ne}^{+}$also shows a positive correlation with $\mathrm{R}_{G}$. For $N_{e}=100 \mathrm{~cm}^{-3}, \mathrm{Ne}^{++} / \mathrm{Ne}^{+}=$ $0.0326+0.0112 \mathrm{R}_{G}$ with extremely little change to this equation at $1000 \mathrm{~cm}^{-3}$. There is more scatter about the fit than for the $\left\langle\mathrm{S}^{++}\right\rangle /\left\langle\mathrm{Ne}^{+}\right\rangle$ratio because the [Ne III] line 

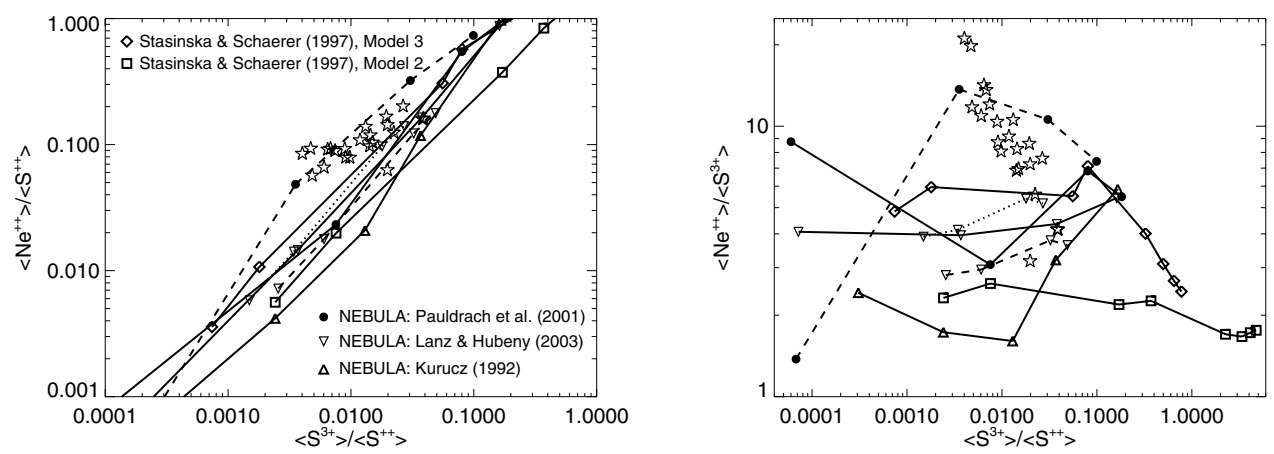

Figure 1. Left panel: Theoretical predictions of the $\left\langle\mathrm{Ne}^{++}\right\rangle /\left\langle\mathrm{S}^{++}\right\rangle$ratio vs. the $<\mathrm{S}^{3+}>/<\mathrm{S}^{++}>$ratio. The line segments connect the points which are the results of nebular models calculated using the ionizing SEDs that are predicted from various stellar atmosphere models as labeled. Two curves show the results from Stasińska \& Schaerer (1997). The rest result from using our photoionization code NEBULA (e.g., Simpson et al. 2004; Rodríguez \& Rubin 2005) changing no other parameter except the SED. For the H II region models calculated with Pauldrach et al. atmospheres, the solid line connects models with dwarf atmospheres and the dashed line connects models with supergiant atmospheres. For the $\mathrm{H}$ II region models calculated with Lanz \& Hubeny atmospheres, the solid line connects models with atmospheres with $\log g=4.0$ and the dotted and dashed lines connect models with atmospheres with $\log g=3.0$ to 3.5 and with Lyman continuum luminosities of $10^{49}$ and $10^{50}$ photons s ${ }^{-1}$, respectively. To compare our data with the models, we need to divide the observed $\mathrm{Ne}^{++} / \mathrm{S}^{++}$ and $\mathrm{Ne}^{++} / \mathrm{S}^{3+}$ ratios by an assumed $\mathrm{Ne} / \mathrm{S}$ abundance ratio. We use the Orion Nebula $\mathrm{Ne} / \mathrm{S}=$ 14.3. The star points (adjusted by the assumed Ne/S) are derived from our line fluxes using $N_{e}$ of $100 \mathrm{~cm}^{-3}$. An $N_{e}$ of $1000 \mathrm{~cm}^{-3}$ will shift these points slightly to the upper right. Right panel: The same as left panel except the ordinate is $\left\langle\mathrm{Ne}^{++}\right\rangle /\left\langle\mathrm{S}^{3+}\right\rangle$. An $N_{e}$ of $1000 \mathrm{~cm}^{-3}$ will shift these points slightly to the bottom right. Both panels dramatically illustrate the sensitivity of the $\mathrm{H}$ II region model predictions of these ionic abundance ratios to the ionizing SED that is input to nebular plasma simulations. The M83 data lie closest to the Pauldrach et al. supergiant locus.

is weaker than either the [Ne II] or [S III] line in these objects. There is a correlation of increasingly higher ionization with $\mathrm{R}_{G}$. This is most likely due to the lower metallicity at larger $\mathrm{R}_{G}$ causing the exciting stars to have a harder ionizing spectrum.

\section{Comparison with models}

The best fit to the data (see Fig. 1) results from the nebular models that utilize the Pauldrach et al. (2001) supergiant stellar atmosphere models (the dashed line connecting filled circles). This result is not sensitive to the range of $N_{e}\left(\sim 100-1000 \mathrm{~cm}^{-3}\right)$ expected for these M83 nebulae.

\section{Acknowledgements}

We acknowledge support from the Spitzer Space Telescope Cycle 1 program 3412.

\section{References}

Kurucz, R.L. 1992, in: B. Barbuy \& A. Renzini (eds.), IAU Symp. 149, Stellar Population of Galaxies p. 225

Lanz, T. \& Hubeny, I. 2003, ApJS 146, 417

Pagel, B.E.J. \& Edmunds, M.G. 1981, ARA\&A 19, 77

Pauldrach, A.W.A., Hoffmann, T.L. \& Lennon, M. 2001, A\& A 375, 161

Rodríguez, M. \& Rubin, R.H. 2005, ApJ 626, 900

Simpson, J.P., Rubin, R.H., Colgan S.W.J., Erickson, E.F. \& Haas, M.R. 2004, ApJ 611, 338

Stasińska, G. \& Schaerer, D. 1997, A\&\&A 322, 615 